\title{
PRÁTICAS DE ENSINO CONTEXTUALIZADAS PARA ALUNOS DO ENSINO FUNDAMENTAL COM DÉFICIT DE ATENÇÃO
}

Aparecida Dias Macedo ${ }^{1}$

Maria do Carmo de Lima²

\section{RESUMO}

Este artigo de revisão bibliográfica narrativa objetivou mostrar que as práticas de ensino com jogos. As atividades práticas estimulam a criatividade, a crítica e a reflexão no processo de ensino e aprendizagem, proporcionando um aprendizado mais significativo na construção dos saberes Ainda, através desses jogos apreendeu-se que é fundamental investigar o conceito de prática pedagógica para atender com qualidade no processo educativo de alunos com diagnóstico de déficit de atenção - TDAH. Aos alunos com histórico de TDAH pretendeu-se despertar o gosto pela aprendizagem de forma mais lúdica e estimular a adoção de estratégias para resolução de problemas matemáticos, desenvolver o raciocínio lógico fazendo uso de jogos com materiais disponíveis na escola, tudo de modo a desenvolver para contribuir no processo de ensinoaprendizagem das disciplinas de Matemática e Ciências. Em relação aos procedimentos metodológicos, o estudo enquadra-se dentro da abordagem da pesquisa de textos, artigos e trabalhos já realizados disponível em meio físico ou eletrônico. Assim, verificou-se que a metodologia tradicional baseada em

\footnotetext{
${ }^{1}$ Graduada em Licenciatura Plena em Matemática pela Faculdade de Filosofia, Ciências e Letras de Alegre/ES; Licenciatura Plena em Geografia pela Universidade Metropolitana de Santos/SP, Especialista Lato Sensu em Educação Especial e Matemática pela Faculdades Integradas de Jacarepaguá; Especialista em Gestão Escolar: S.E; O.E; I.E pela Universidade Castelo Branco; aluna do Curso de Mestrado Stricto Sensu pela Faculdad Interamericana de Ciencias Sociales- FICS; atua como professora na rede municipal de Muniz Freire/ES;

2 Graduada em Direito pela Faculdade de Direito do Vale do Rio Doce Governador Valadares/MG; Pós graduada em Direito Público pela Faculdade de Direito Monsenhor Messias, Sete Lagoas/MG; formação em Tutoria curso EAD - Universidade Federal de Ouro Preto/MG; formação em Tutoria cursos Ead Unviersidade Federal de São João Del-Rei/MG; Acadêmica do Curso de Pós-Graduação Stricto Sensu em Ciências da Educação pela Faculdad Interamericana de Ciencias Sociales-FIC; Advogada, Especialista em Direito Público, Direito Médico, Mediadora de Conflitos pelo NUPEMEC da EFEJ/TJMG; professora regente rede municipal e particular de ensino. marialimaadv@gmail.com
} 
explicação do professor, aplicação de questionários de fixação é menos significativa que a metodologia baseada na aplicação de atividades práticas. Os estudos do acervo bibliográfico contido neste artigo apontam que a prática aliada com a teoria ensejou uma aprendizagem mais efetiva e estimuladora do aluno para realização de tarefas e outras atividades escolares tornando-o mais ativo, mais autônomo durante as práticas escolares a ele proposta em sala de aula.

PALAVRAS-CHAVE: Aluno. Transtorno de Déficit de Atenção e Hiperatividade. TDAH. Educadores. Estratégias. Ensino.

\section{ABSTRACT}

This narrative bibliographic review article aimed to show that teaching practices with recyclable materials available in the environment in which the student is inserted. Practical activities stimulate creativity, criticism and reflection in the teaching and learning process, providing more meaningful learning in the construction of knowledge. Through these games, it was learned that it is essential to investigate the concept of pedagogical practice in order to attend with quality in the classroom. educational process of students diagnosed with attention deficit - ADHD. The aim of students with a history of ADHD was to arouse a taste for learning in a more playful way and encourage the adoption of strategies for solving mathematical problems, developing logical reasoning using recyclable materials available at school through games, all in order to develop to contribute to the teaching-learning process of the subjects of Mathematics and Science. Regarding the methodological procedures, the study fits within the approach of searching for texts, articles and works already carried out, available in physical or electronic medium. Thus, it was found that the traditional methodology based on the teacher's explanation, application of fixation questionnaires is less significant than the methodology based on the application of practical activities. The studies in the bibliographic collection contained in this article show that the practice combined with the theory led to a more effective 
and stimulating learning for students to perform tasks and other school activities, making them more active, more autonomous during the school practices proposed to them in class. of class.

KEYWORDS: Student. Attention Deficit Hyperactivity Disorder. ADHD. Educators. Strategies. Teaching.

\section{RESUMEN}

Este artículo de revisión bibliográfica narrativa tuvo como objetivo mostrar que las prácticas de enseñanza con juegos. Las actividades prácticas estimulan la creatividad, la crítica y la reflexión en el proceso de enseñanza y aprendizaje, proporcionando un aprendizaje más significativo en la construcción del conocimiento .A través de estos juegos, se aprendió que es esencial investigar el concepto de práctica pedagógica para asistir con calidad en el aula o proceso educativo de estudiantes diagnosticados con trastorno por déficit de atención TDAH. El objetivo de los estudiantes con antecedentes de TDAH era despertar el gusto por el aprendizaje de una manera más lúdica y alentar la adopción de estrategias para resolver problemas matemáticos, desarrollar el razonamiento lógico através de juegos utilizando materiales disponibles en la escuela, todo para desarrollar para contribuir al proceso de enseñanza-aprendizaje de las asignaturas de Matemáticas y Ciencias. En cuanto a los procedimientos metodológicos, el estudio se ajusta al enfoque de investigación de textos, artículos y trabajos ya realizados, disponibles en medios físicos o electrónicos. Por lo tanto, se encontró que la metodología tradicional basada en la explicación del profesor, la aplicación de cuestionarios de fijación es menos significativa que la metodología basada en la aplicación de actividades prácticas. Los estudios de la colección bibliográfica contenida en este artículo indican que la práctica combinada con la teoría condujo a un aprendizaje más efectivo y estimulante para que los estudiantes realicen tareas y otras actividades escolares, haciéndolos más activos, más autónomos durante las prácticas escolares propuestas en clase. 
PALABRAS CLAVE: Estudiante. Desorden hiperactivo y deficit de atencion. TDAH Educadores Estrategias. Enseñando.

\section{INTRODUÇÃO}

As autoras Blanca Susana Guevara Werlang e Margareth da Silva Oliveira (2006, P.108) conceituam o transtorno do déficit de atenção/hiperatividade (TDAH), vulgarmente conhecido apenas como hiperatividade da seguinte forma:

O TDAH, segundo critérios diagnósticos da American Psychiatric Association - DSM - IV (APA, 1994), é um transtorno mental que consiste em um padrão persistente de desatenção e/ou hiperatividade e impulsividade.

Segundo Neves, (2005, p.8): a hiperatividade em si não é uma doença e, geralmente, um sintoma de algum distúrbio como TDAH (Transtorno de Déficit de Atenção e Hiperatividade).

É sabido que a escola é o primeiro grande grupo que a criança irá conviver fora da família. Na escola a criança construirá grande parte de sua história. No período pré-escolar, os sintomas de hiperatividade e desatenção podem não ser facilmente identificados pelo fato de as atividades serem mais dinâmicas, a atenção do professor ser mais individualizada e haver menos expectativa em relação ao desempenho de todos.

(...) A partir da alfabetização, as crianças começam a participar de atividades que exigem atenção por um período maior e surgem novas exigências quanto ao comportamento. Começa a se exigir que a criança permaneça mais tempo sentada em sala de aula, o conteúdo didático é aprofundado e se torna necessário a responsabilidade com deveres de casa. (MATTOS, 2003, p.28)

Também é corrente o entendimento que tanto a família quanto o aluno esperam da escola um lugar diferenciado e preparado para receber crianças TDAH com 
atendimento individualizado, mas o sistema tradicional de ensino ainda despreparado para lidar com os portadores desse transtorno em pouco ou nada se adaptando ao aluno portador de TDAH, esperando que o aluno se adeque ao nível de exigências e de conteúdos pré-estabelecidos pela escola podendo desencadear choques que provavelmente se transformarão em problemas na vida dos indivíduos com TDAH.

Para Vygotsky, o desejo de brincar ajuda a desenvolver a linguagem e os significados e sem ele "seria muito mais áspera a transposição entre os significados e os recursos significantes" (ANTUNES, 2003, p. 19).

Ainda Antunes, a criança que brinca está desenvolvendo sua linguagem oral, seu pensamento associativo, suas habilidades auditivas e sociais, construindo conceitos de relações espaciais e se apropriando de relações de conservação, classificação, seriação, aptidões visuo espaciais e muitas outras. ( 2003, p. 19)

Segundo Smole, Diniz e Milani (2007): Os jogos auxiliam o desenvolvimento de habilidades como observação, análise, levantamento de hipóteses, busca de suposições, reflexão, tomada de decisão, argumentação e organização. Estas habilidades desenvolvem-se porque ao jogar os alunos tem a oportunidade de resolver problemas, investigar e descobrir a melhor jogada; refletir e analisar as regras; estabelecendo relações entre os elementos do jogo e os conceitos matemáticos. Enfim, o jogo possibilita uma situação de prazer e aprendizagem significativa nas aulas de matemática.

$\mathrm{Na}$ sua obra multicitada, Antunes traz estudiosos da psique humana e da educação como Sigmund Freud, Henri Wallon, Caillois, Vygotsky e Jean Piaget que defendem as brincadeiras e os jogos como eficazes instrumentos pedagógicos. Para Freud, canalizam a energia (libido) das crianças construindo "processos de sublimação saudáveis e identificadores", propiciando "o afeto e a sociabilidade, dando voz aos sonhos infantis" (ANTUNES, 2003, p. 18)

Nesse passo, a boa escola não é necessariamente aquela que possui uma quantidade enorme de caríssimos jogos eletrônicos ou jogos ditos educativos, mas que disponha de uma equipe de educadores que saibam utilizar a reflexão que o jogo desperta, saibam fazer de simples objetos naturais uma oportunidade 
de descoberta e exploração imaginativa. Uma caixa de fósforo, uma lupa e uma fita métrica em mãos de uma verdadeira educadora infantil valem bem mais que uma fantástica coleção de brinquedos eletrônicos que emitem luzes e sons e que, por se apresentarem perfeitos demais roubam espaço à imaginação. (ANTUNES, 2003, p. 31)

Justificou-se este estudo acrescentar oportunidades de aprendizagem para 0 aluno portador de TDAH visando também estimular convivência social e desenvolver habilidades através de práticas pedagógicas consistentes em jogos.

Elegemos como hipótese apontar quais as estratégias pedagógicas que os educadores utilizam na prática docente objetivando trabalhar questões de sociabilidade e de aprendizagem com seus alunos portadores de TDAH a partir de práticas pedagógicas aliadas ao conhecimento teórico.

Este estudo teve como objetivo esclarecer o alcance das práticas pedagógicas contextualizadas utilizando-se de jogos para desenvolver o raciocínio lógico e contribuir no processo de ensino-aprendizagem de alunos com hiperatividade.

\section{REFERENCIAL TEÓRICO}

De acordo com Ronca (1989, p. 27) "O movimento lúdico, simultaneamente, torna-se fonte prazerosa de conhecimento, pois nele a criança constrói classificações, elabora sequências lógicas, desenvolve o psicomotor e a afetividade e amplia conceitos das várias áreas da ciência”.

Segundo Espírito Santo (2002, p. 35), "o trabalho do educador é fazer com que o aluno tome consciência de seu potencial criador e destruidor", e do que suas ações terão como resultado.

Os jogos que possuem regras permitem que a criança, além de ter seu desenvolvimento social quanto aos limites, possa participar saber ganhar, perder, melhorar seu desenvolvimento cognitivo, e possibilita a oportunidade para a criança saber onde está, o motivo e o tipo de erro que cometeu, tendo chance de refazer, naquele momento, da maneira correta. (BENCZIK, 2000).

Segundo (STROH, 2010): 
[...] Jogo com regras: a criança deverá submeter-se às regras e normas, onde poderá desenvolver suas habilidades, seu raciocínio, autoimagem, tolerar frustrações, saber ganhar ou perder, saber esperar sua vez, planejar uma situação, aprender a ouvir, etc. Uso de sucata: estimula a criatividade ao poder criar e formar novos materiais $[\ldots]$

\section{PROCEDIMENTOS METODOLÓGICOS}

Foram feitos estudos em várias fontes bibliográficas sobre o tema título deste artigo, que trouxeram com profundidade a importância da coexistência da teoria e da prática para melhor aprendizagem dos alunos com comprovado déficit de atenção. Esses estudos abordaram os desafios diários de professores para a correta utilização de jogos nas atividades docentes em sala de aula.

Neste estudo foram selecionadas fontes de pesquisa bibliográficas em meio impresso e eletrônico estes últimos constituídos em artigos publicados na web, extraídos das páginas da Scielo, Ciência \& Educação e Espaço Acadêmico que tão bem contribuíram para deitar mais luzes sobre o tema "Práticas de Ensino Contextualizadas para alunos do ensino fundamental com déficit de atenção".

\section{RESULTADOS ESPERADOS E DISCUSSÕES}

Para Marcelo Giordan (1999, p.43) em históricos de Aristóteles (1999, p.1-303) "quem possua a noção sem a experiência, e conheça o universo ignorando o particular nele contido, enganar-se-á muitas vezes no tratamento". Isso comprova que a prática ou o experimento é indispensável para a aplicação do conhecimento.

Assim, o conhecimento científico está associado à experimentação, à pratica das do conhecimento empírico. Para Schnetzler e Aragão (1995, p.27), Krasilchik 
(1987, p. 82), em Giordan (1999, p.45), "a partir dos anos 60, a cultura de pesquisa na área de Ciências passou a influenciar os programas de educação científica, sob a influência da psicologia cognitiva e da epistemologia estruturalista, além de outras áreas do conhecimento".

$\mathrm{Na}$ história da Educação brasileira ao aluno era solicitada a memorização de conteúdo didático, além da repetição de matérias para realizar exercícios, questionários, provas, as quais tinham como objetivo principal a aprovação em séries seguintes. Registra-se que "é interessante ressaltar que as atividades práticas no Brasil começaram a ser realizadas na década de 30. ( MARANDINO, 2003, p.3).

Toda criança, assim como o adulto tem suas limitações. Daí a criança tem o seu tempo e o seu limite. E, os portadores de TDAH não são diferentes, porque possuem um problema. E quem de nós não tem problemas? Eles não são mais ou menos inteligentes por isso. Pelo contrário "a grande maioria das crianças e adolescentes com este problema têm inteligência na faixa normal para sua idade e tentam esforçar-se ao máximo para prestar atenção e parar quietas". (ROHDE e BENCZIK, 1999, p. 45)

De acordo com Carvalho (2009 p.52), "recentes pesquisas sobre o ensino de ciências relatam que com aulas teóricas e práticas os alunos se interessam mais pelas aulas e acabam com a 'visão deformada" sobre o ensino de ciências'. Conclui-se que a associação entre aulas teóricas e práticas é capaz de ampliar e melhorar a qualidade pedagógica dos processos de ensino e aprendizagem no cotidiano escolar.

Para Tardif (2002, p.237), "as atividades práticas permitem aprendizagens que a aula teórica, apenas, não permite, sendo compromisso do professor, e também da escola, dar esta oportunidade para a formação do aluno". Compreendemos então que, de acordo com a necessidade do planejamento realizado pelo professor, a realização de atividades práticas se faz necessária para a efetiva aprendizagem do aluno. 
Borges (2002) relata que a meta principal das atividades práticas em sala de aula é mostrar ao aluno o que aprendeu em suas aulas teóricas, para que, através das aulas práticas, ele possa compreender como de fato ocorre o conteúdo trabalhado na aula teórica. Ele relata que:

Não se pode deixar de reconhecer alguns méritos deste tipo de atividade. Por exemplo, a recomendação de se trabalhar com pequenos grupos, o que possibilita a cada aluno a oportunidade de interagir com as montagens e instrumentos específicos, enquanto divide a responsabilidade e ideias sobre o que devem fazer e como fazê-lo. Um outro é o caráter mais informal do laboratório, em contraposição à formalidade das demais aulas. (BORGES, 2002 p.5)

Entendeu-se que o modelo tradicional de ensino dominado pela transmissão do conhecimento evoluiu para atrelar-se à atividade prática trazem outro significado ao aprendizado.

Assim, a atividade prática pressupõe participação do aluno em uma situação de ensino e aprendizagem em que se utiliza ou requer a análise e reflexão sobre dados primários da natureza.

Peres (2013,p.70) argumenta que o "jogo é o meio de aprendizagem pelo qual a criança investiga, explore e descobre o mundo que o rodeia, pois conhecendo o mundo, a criança vai conhecendo a si mesma."

A busca destas situações leva o professor a criar tarefas que propiciem aos estudantes experiências físicas e lógico-matemáticas, para as quais se faz necessário integrar questões, leituras, debates, que não constituem a atividade prática em si, mas a complementam. (ANDRADE; MASSABNI, 2011).

As atividades contextualizadas para alunos com DTAH têm contribuído para o aprendizado dos conteúdos curriculares. Constatou-se ainda que o interesse dos alunos ocorreu devido à metodologia do uso de jogos como meio facilitador da aprendizagem. 


\section{CONSIDERAÇÕES FINAIS}

A partir da pesquisa bibliográfica onde se relatou sobre a importância de se associar o conhecimento teórico às atividades práticas nas salas de aula para alunos com déficit de atenção e de aprendizagem nas series do Ensino Fundamental, através do olhar diferenciado de docentes permitiu concluir que o trabalho há de ser conjunto com atividades lúdicas prazerosas respeitando as etapas de desenvolvimento intelectual dos alunos como seu rendimento nas disciplinas, sua organização na sala e com seu material, interesse na matéria, comportamento em sala de aula e nas atividades fora da sala, culminando na aprendizagem formal das disciplinas de ciências e matemática, além de seu relacionamento com os colegas e professores na construção dos saberes.

A utilização de metodologias criativas e inovadoras no cotidiano do ensino escolar, a partir da proposta pedagógica para o ano letivo em cada série ou ano e em cada unidade didática a fim de se incentivar o gosto dos alunos do Ensino Fundamental pela aprendizagem porque é nesta fase escolar que eles estão vivenciando descobertas também em seus estudos, e as disciplinas de Ciências e de Matemática se apresentam como instigadoras, bem sugestivas, de sempre que necessário, se aplicada com práticas que tornem mais atraentes a disciplina o êxito, o avanço na qualidade pedagógica acontecer introduzindo atividades em sala de aula que possam gerar aprendizagens em seu alunado no cotidiano escolar.

\section{REFERÊNCIAS}

ANDRADE, Marcelo Leandro Feitosa de; MASSABNI, Vânia Galindo. O Desenvolvimento de Atividades Práticas na Escola: um desafio para os professores de Ciências. Bauru/ SP. 2011. Ciência \& Educação (Bauru). Vol. 17. № 4.

ANTUNES, Celso. O Jogo e a Educação Infantil: falar e dizer, olhar e ver, escutar e ouvir. $2^{\mathrm{a}}$ ed. Fascículo 15. Petrópolis, RJ: Vozes, 2003. 
ARISTÓTELES. Metafísica. São Paulo: Editora Abril, 1979. Livro A, cap. I. (Coleção Os Pensadores) Orig. do século IV a.C

BENCZIK, Bellini Peroni Benczik. Transtorno de Déficit de Atenção/Hiperatividade. Atualização Diagnóstica e terapêutica. Um guia de orientação para profissionais. São Paulo: Casa do Psicólogo. 2006.

BORGES, Tarciso; Caderno Brasileiro de Ensino de Física. 2002. Disponível em: $\begin{array}{llll}\text { Acesso junho de } & \text { 2020, }\end{array}$ https://periodicos.ufsc.br/index.php/fisica/article/view/6607/6099

CARVALHO, Anna Maria Pessoa de. Ensino de Ciências: unindo a pesquisa e a prática. São Paulo, 2009. Pioneira Thomson.

ESPÍRITO SANTO, Ruy Cezar do. Desafios na formação do educador: Retornando $\mathrm{o}$ ato de educar. Campinas, (SP): Editora Papirus, 2002.

GIORDAN, Marcelo. O papel da Experimentação no ensino de Ciências. Belo Horizonte, Novembro de 1999. Pesquisa no Ensino de Química. Revista Química Nova na Escola. № 10.

KRASILCHIK, M. O professor e o currículo de ciências. São Paulo, EPU/ Edusp, 1987.

MARANDINO, Martha, A Prática de Ensino nas Licenciaturas e a Pesquisa em Ensino de Ciências Questões Atuais. 2003. Disponível em: https://periodicos.ufsc.br/index.php/fisica/article/view/6544/6034 Acesso em 15 de jun.2020

MATTOS, Paulo. No Mundo da Lua: Perguntas e respostas sobre o transtorno do déficit de atenção com hiperatividade em crianças, adolescentes e adultos. $4^{a}$ ed.. São Paulo: Lemos Editorial, 2003. 
NEVES, I. F.; SCHOCHAT, E. Maturação do processamento auditivo em crianças com e sem dificuldades escolares. Pró-Fono Revista de Atualização Científica. Barueri, SP set/dez.2005. v.17.n.3, disponível em https://www.scielo.br/pdf/pfono/v17n3/v17n3a04.pdf, acessado em 15 de jun.2020.

PERES, Clarice. TDA-H (Transtorno de Déficit de Atenção e Hiperatividade): da Teoria à Prática: manual de estratégias no âmbito familiar, escolar e de saúde. Rio de Janeiro: Wak Editora, 2013. SILVA, Ana Beatriz Barbosa. Mentes Inquietas: TDAH: desatenção, hiperatividade e impulsividade. Rio de Janeiro: Objetiva, 2009.

PHELAN, T. W. TDA/TDAH. Transtorno de Déficit de Atenção e Hiperatividade: Sintomas, Diagnósticos e Tratamentos. Crianças e Adultos São Paulo: M. Books do Brasil Editora, 2005.

PSYCHIATRIC ASSOCIATION, AP, editor. Diagnostic and statistical manual of mental disorders: DSM-IV-TR. Americam Psychiatric Pu

RONCA P.A.C. A aula operatória e a construção do conhecimento. São Paulo: Edisplan, 1989.

SCHNETZLER, R. P. \& ARAGÃO, R. M. R. Importância, sentido e Contribuições de pesquisa para o ensino de Química. Belo Horizonte, 1995. Química Nova Escola, № 1, p. 27- 31.

SMOLE,Kátia Stocco; DINIZ, Maria Ignez; MILANI, Estela. Cadernos do Mathema: Jogos de Matemática de 6ํㅜ a 9ำ ano. Porto Alegre: Artmed, 2007.

STROH, Juliana Bielawski. TDAH - diagnóstico psicopedagógico e suas intervenções através da Psicopedagogia e da Arte terapia. Construção Psicopedagógica, São Paulo, Instituto Sedes Sapientiae, v. 18, n. 17, p. 83105 , dez. 2010.

Disponível em: 
https://bell.unochapeco.edu.br/revistas/index.php/pedagogica/article/viewFile/37 52/2123 Acesso em: 15 jun.2020.

TARDIF, M. Saberes docentes e formação profissional. Petrópolis: Vozes, 2002. 3를ição.

WERLANG, Blanca Susana Guevara; OLIVEIRA, Margareth da Silva. (orgs.). Temas em Psicologia Clínica. São Paulo: Casa do Psicólogo, 2006 